\title{
ARIA
}

\section{La Commission européenne face à l'efficacité et l'équité des systèmes éducatifs européens}

\author{
MARC DEMEUSE \\ Institut d'Administration scolaire \\ Université de Mons-Hainaut \\ Académie universitaire Wallonie - Bruxelles \\ Place du Parc 18, B-7000 Mons (Belgique)
}

\author{
ARIANE BAYE \\ Unité d'Analyse des Systèmes et des Pratiques d'Enseignement \\ Université de Liège \\ Académie universitaire Wallonie - Europe \\ Boulevard du Rectorat 5 (Bât. B32), B-4000 Liège (Belgique)
}

L

a communication de la Commission européenne, intitulée Efficacité et équité des systèmes européens d'éducation et de formation (2006), propose de considérer le double défi qui s'offre aux systèmes d'enseignement et de formation européens : assurer compétitivité et cohésion sociale. Elle pose d'emblée le cadre dans lequel ce défi doit prendre place : une conjoncture de restriction des dépenses publiques et quatre pressions importantes : la mondialisation, la démographie, l'évolution rapide de la nature du marché du travail et l'innovation technologique. Dès le deuxième paragraphe, se dessine une approche résolument économique qui se maintient dans l'ensemble du texte, élaboré sur la base d'un document interne Commission staff working document (SEC-2006 1096), lui-même préparé par un texte produit par les experts du "Réseau Européen d'Experts en Économie de l'Éducation” (EENEE) (Wößmann \& Schültz 2006). Cette orientation se traduit dans l'approche des deux concepts centraux du texte : l'équité et l'efficacité. 
L'équité est définie comme "le degré auquel les individus peuvent bénéficier de l'éducation et de la formation, en matière de possibilités, d'accès, de traitement et de résultats". Cette définition mêle, sans le préciser, différentes conceptions qui mériteraient plus d'attention qu'une note en bas de page (note 2, 2). Ces différentes conceptions, bien décrites par exemple dans les travaux du Groupe Européen de Recherche sur l'Équité des Systèmes Éducatifs (EGREES 2005), reposent sur des exigences différentes: égalité des chances d'une part, égalité effective d'accès, de traitement ou de résultats (internes ou externes) d'autre part. Si la première conception renvoie à l'idée d'une égalité potentielle (avoir virtuellement les mêmes chances d'obtenir un diplôme ou un autre bien éducatif), les trois autres concernent des égalités effectives -pouvoir accéder à un même service éducatif (l'enseignement supérieur, par exemple), bénéficier d'un traitement égal (enseignants ou locaux de qualité égale), obtenir des résultats égaux (par exemple, maîtriser les compétences de base). Poursuivant sa définition, la communication de la Commission précise qu'un système est défini comme équitable, d'une part si "les résultats de l'éducation et de la formation sont indépendants du milieu socio-économique et d'autres facteurs conduisant à un handicap éducatif" et, d'autre part, si "le traitement reflète les besoins spécifiques des individus en matière d'apprentissage". La conception de l'équité éducative retenue par la Commission, à savoir l'indépendance entre le statut socioéconomique et les résultats de l'éducation, correspond au dénominateur commun sur lequel s'accordent la plupart des théoriciens de la justice (Meuret 2001). La Commission ne retient cependant pas la notion de "seuil" introduite par le GERESE (EGREES 2005), qui précise qu'en deçà d'un certain seuil de compétences, les individus ne sont pas suffisamment armés pour poursuivre leur scolarité et faire face aux demandes de compétences nécessaires pour s'insérer de manière satisfaisante dans la société civile et sur le marché de l'emploi. La suite de la note explicative est par contre assez curieuse : "Les injustices fondées sur l'appartenance à l'un ou l'autre sexe ou à une minorité ethnique, un handicap, des disparités régionales, etc., ne forment pas le thème principal du présent document, mais doivent être prises en considération dans la mesure où elles contribuent aux désavantages socioéconomiques globaux". Il semble donc que la Commission, dans sa communication, ne souhaite traiter que des inégalités d'origine socioéconomique, à l'exclusion de toute autre source qui ne conforterait pas cette origine. Il est pourtant à la fois difficile d'isoler les causes, notamment lorsqu'elles entrent en interaction, et regrettable de ne pas prendre en compte des facteurs aussi déterminants que ceux évoqués par la Commission elle-même. Le sexe, l'appartenance ethnique ou un handicap constituent autant de conditions auxquelles les individus ne peuvent échapper, ce qui rend les inégalités dont ils sont victimes particulièrement injustes. La situation de handicap, lorsqu'elle frappe les facultés mentales, peut néanmoins, assez classiquement, 
être traitée de manière distincte puisqu'elle ne permet pas aux individus d'atteindre les mêmes objectifs cognitifs, malgré l'accroissement des moyens éducatifs. Il reste à déterminer ce qu'il est juste de consacrer à la réalisation d'autres objectifs que les objectifs communs pour cette population spécifique qui ne peut atteindre les objectifs de compétitivité et d'excellence.

Un système équitable est donc un système qui ne produit pas d'inégalités injustes. Les récents travaux du GERESE (EGREES 2005, Baye, Demeuse, Monseur $\&$ Goffin 2006) expliquent que l'équité est une notion normative intimement liée à la notion de justice. Il s'agit donc, pour les États-membres, de définir les inégalités d'éducation injustes. Remplacer le terme égalité par celui d'équité ne dispense pas de se prononcer sur le modèle de justice, ce que la note de la Commission passe sous silence.

La Commission (note 3, 2) définit l'efficacité comme "la relation, dans un processus, entre les moyens mis en œuvre et les résultats obtenus”. Il s'agit donc davantage d'une mesure de l'efficience -atteinte de résultats à moindre coûtque de l'efficacité au sens pédagogique -atteinte des objectifs (sur la distinction entre efficacité et efficience, Reezigt 2001, 2-4). Selon la note, un "système est efficace si les moyens mis en œuvre donnent un résultat maximal". Cette approche est celle de la stratégie de Lisbonne : il ne s'agit pas tant d'assurer à tous un niveau de vie satisfaisant en regard de critères définis que de devenir l'économie de la connaissance la plus compétitive (Conseil européen 2000). Cette approche utilitariste visant le meilleur résultat moyen possible, au risque de laisser sur le côté une part importante de la population- conforte la nécessite de s'intéresser, au moins sous couvert d'équité, à la frange des individus sous le seuil et pas uniquement aux écarts entre les plus favorisés et ceux qui le sont moins.

En termes d'équité pédagogique, les objectifs de Lisbonne pour les Étatsmembres de l'Union européenne pourraient être considérés comme le minimum à atteindre. Les rapports de suivi du processus de Lisbonne (Commission of the European Communities 2004 et sq.) peuvent dès lors être lus comme autant d'évaluations de l'efficacité pédagogique des systèmes éducatifs européens. L'évaluation de leur efficience (économique) supposerait qu'on s'entende sur un rapport idéal entre des investissements et des résultats ou, du moins, que les entrées (inputs) soient prises explicitement en compte, en regard des résultats. Or il n'y a pas encore d'indicateurs allant dans ce sens et on constate que la Commission ne fait pas de proposition en ces termes, ce qui semble logique dans la mesure où les objectifs établis à Lisbonne prévoient d'augmenter les dépenses, y compris privées (Communication de la Commission janvier 2003). Les rapports sur lesquels se fondent les propositions de la Commission, même s'ils utilisent le terme "efficacité", confondent cette notion avec celle d'efficience, puisque les évaluations sont réalisées par rapport aux données cognitives, mesurant les acquis des élèves (donc le rendement "cognitif" des systèmes éducatifs), et non en fonction 
des "inputs" (en termes d'argent, de charges enseignantes, de temps...) introduits dans les différents systèmes éducatifs. Si la littérature récente dans le domaine des sciences de l'éducation montre bien que l'efficacité et l'équité peuvent se renforcer mutuellement, une telle analyse n'a pas encore été effectuée par la Commission sur le lien entre efficience et équité.

\section{Enseignement préprimaire : se concentrer sur l'apprentissage à un âge précoce}

T es propositions de la Commission se fondent en grande partie sur le modèle théorique proposé par Cunha, Heckman, Lochner \& Masterov (2005). Ce
modèle économique du développement de l'enfant postule que les compétences innées ou acquises à un certain âge sont le socle sur lequel se greffent les apprentissages ultérieurs. Partant de ce principe que le bon sens ne saurait contredire, les auteurs affirment que, plus les investissements sont précoces, meilleur sera leur taux de retour, puisque les fruits de ces investissements durent toute la vie. À l'inverse, des investissements ultérieurs ont moins de retour, puisqu'effectifs sur une plus courte période, et doivent être plus conséquents, puisque, plus l'enfant grandit, plus il est difficile de remédier à des lacunes accumulées depuis la petite enfance.

Aussi séduisante que puisse paraître cette théorie "balistique", elle peut être remise en cause sur plusieurs points. D'abord, elle ne prend pas en compte le fait que les difficultés d'apprentissage puissent survenir relativement tard dans le processus de développement de l'enfant ou de l'adolescent, sans qu'il soit aisé de rattacher ces difficultés à un déficit lors des étapes antérieures. Les difficultés constatées à un moment ou à un niveau scolaire donné pourraient ainsi être trop facilement et systématiquement attribuées au niveau scolaire précédent. Ceci peut avoir pour effet de déresponsabiliser les intervenants éducatifs ultérieurs, tout en les privant d'une attention et d'outils nécessaires pour prévenir les décrochages scolaires et y remédier, notamment dans le secondaire. En second lieu, le modèle de Cunha $\&$ al. (2005) repose sur des données empiriques recueillies aux États-Unis, peu convaincantes pour les pays européens. Ensuite, elles ne conviennent pas pour tester le modèle général des auteurs, puisqu'elles ne rendent pas compte du développement des compétences mesurées dans le préprimaire et dont on aurait suivi l'évolution jusqu'au moins la fin de la scolarité secondaire (pas de suivi longitudinal long).

Enfin, pour saisir la portée du modèle proposé, il faut connaitre la spécificité de l'enseignement préprimaire aux États-Unis. Les données internationales sur la fréquentation du préprimaire montrent que les traditions nationales divergent : alors que le taux de participation des enfants de moins de 4 ans est de 
52,9\% aux États-Unis, il est en moyenne de 73,5 \% dans les pays membres de l'Union européenne (EU-19, d'après OECD 2006, 266). Cette moyenne élevée s'explique à la fois par une implantation de longue date du préprimaire dans certains États-membres (Belgique, France, Italie, Espagne) et par de gros efforts de développement ces dernières décennies dans l'Union européenne, comme l'indiquent les données d'Eurydice sur l'évolution (1979-2002) des taux de fréquentation dans les établissements préprimaires à finalité éducative par les enfants de 4 ans $(2005,133)$.

L'affirmation selon laquelle l'enseignement préprimaire affiche le rendement le plus élevé sur les plans des résultats obtenus et de l'adaptation sociale des enfants ne se fonde pas sur des données empiriques dans les États-membres de l'Union européenne. Étant donné la diversité des taux nationaux de fréquentation du préprimaire, une politique commune de l'Union européenne ne peut être uniformément valable. Il faut souligner que les travaux évaluant l'impact des structures de garde précédant la scolarité obligatoire sont discordants. Si l'impact est globalement positif, il l'est plus sur l'adaptation sociale que sur le langage (Duru-Bellat 2003). Dans sa synthèse sur les inégalités sociales à l'école, DuruBellat indique aussi que les écarts sociaux peuvent s'accrôitre légèrement par la fréquentation de l'école maternelle, car, tous les enfants tirant profit d'une scolarisation précoce, les plus favorisés en profitent encore davantage.

Dans les pays de l'Union européenne, les données de la récente étude PIRLS (Mullis \& al. 2003) portant sur les compétences en lecture des élèves du primaire, peuvent être convoquées pour étayer le débat. S'il ne s'agit pas de données longitudinales permettant de répondre à l'affirmation de la Communication, les questionnaires de contexte utilisés permettent de corréler les résultats en lecture et la durée de fréquentation du préprimaire. En moyenne, pour les pays de l'Union participant à l'enquête, la corrélation est inexistante $(-0.14)$ entre la proportion d'enfants ayant fréquenté le préprimaire plus de deux ans et les résultats moyens en lecture en primaire. Pourtant, la fréquentation de structures préprimaires semble bénéfique aux plus faibles, puisque le taux de fréquentation des enfants de 4 ans de dispositifs d'enseignement préprimaires à finalité éducative (Eurydice 2002, 133) est positivement corrélé $(0.41)$ au taux d'élèves atteignant au moins le 25e percentile en lecture (Mullis \& al. 2003, Eurydice 2002).

La faiblesse de l'argumentaire de la Commission ne doit cependant pas faire oublier l'importance de l'enseignement préscolaire. Elle souligne surtout l'insuffisance de la recherche sur les bénéfices à long terme de l'enseignement préprimaire dans l'Union européenne. Les recommandations de la Commission gagneraient à intégrer les politiques déjà mises en œuvre dans les États-membres. À ce titre, l'étude portant sur l'éducation et l'accueil des enfants avant l'âge de la scolarité obligatoire réalisée par l'OECD (2001) relève des politiques convergentes. Le rapport note une tendance à l'extension des services vers au moins 
deux ans de services subventionnés gratuits pour tous avant la scolarité obligatoire. La centration sur ce point des mesures financières proposées par la Commission, ne ferait que renforcer des politiques en cours, au détriment de la scolarité obligatoire pour laquelle la Commission préconise peu. Le rapport de l'OCDE (2001) indique que les préoccupations actuelles privilégient la qualité des services, en raison du manque de cohérence des politiques et des services visant l'éducation et l'accueil des jeunes enfants, du manque de formation de certains personnels, de la tendance pour les familles à faibles revenus à avoir des services moins bons.

Plus qu'une attribution prioritaire des ressources, il faut chercher, pour le préprimaire, plus d'efficience via la coordination des politiques et des services et affecter les moyens disponibles à la formation initiale et continuée des personnels, l'amélioration de leur statut et salaires, de meilleurs services pour les enfants les moins favorisés, passant notamment par le travail avec les familles. La définition de documents cadres et d'objectifs pédagogiques centrés sur le développement global de l'enfant devrait permettre de fixer les standards et d'évaluer leur atteinte pour un meilleur pilotage des systèmes d'éducation de la petite enfance. Le rapport de l'OCDE (2001) met aussi en garde contre des mesures décentralisées qui ne limiteraient pas les variations dans l'accès et la qualité des services. Enfin, il pointe la nécessité d'une harmonisation internationale de recueil de données concernant ce niveau.

La nécessité d'investir en faveur des enfants provenant des familles les plus défavorisées est reconnue. Ce constat appelle cependant une réflexion sur la manière d'aider les populations fragilisées, sans les stigmatiser ou les culpabiliser quand, malgré l'aide accordée, elles ne parviennent pas aux résultats escomptés. Les politiques d'éducation prioritaires peuvent parfois se révéler décevantes, pour de multiples raisons, dont le fait qu'elles ne disposent pas toujours réellement des moyens en principe accordés, notamment, parce que les enseignants les plus aguerris sont affectés ailleurs ou choisissent des établissements fréquentés par des élèves moins fragilisés. Un autre handicap provient de l'absence d'évaluations sérieuses prenant en compte à la fois les problèmes en amont (ciblage des publics, définition et sélection des programmes, affectation des moyens humains et matériels...) et la manière dont sont mises en place, sur le terrain, ces actions (Slavin \& Fashola 1998, Wang, Haertel \& Walberg 1998).

\section{Enseignement primaire et secondaire}

T a communication de la Commission réserve une place relativement limitée L à l'étude de l'efficacité et de l'équité lors de la scolarité obligatoire qui devrait être l'objet de toute sa vigilance. En effet, une partie des enfants, notam- 
ment ceux des milieux les moins favorisés, n'a effectivement accès qu'à la scolarité obligatoire et la recherche a montré que des structures et des pratiques pédagogiques différentes peuvent produire des résultats différents, tant en termes d'efficacité que d'équité (Elley 1992, Mullis \& al. 2003, 2004a, 2004b).

Pour la scolarité primaire, la Commission ne mentionne aucune analyse, ne prévoit aucune mesure. Or les données d'études internationales montrent que les pays divergent déjà, tant en termes de rendement moyen que d'inégalités sociales, entre les sexes ou de proportion d'élèves atteignant un certain seuil de compétences, dès le primaire.

À cet égard, on pourrait mettre en parallèle le modèle cumulatif et multiplicateur des compétences acquises proposé par la Commission avec un modèle cumulatif et multiplicateur des inégalités. Ainsi par exemple, l'effet du milieu social sur le rendement scolaire, qui peut être ténu en début de scolarité et sur une année, est incorporé progressivement au niveau scolaire, qui est l'ingrédient principal de la progression au degré ultérieur. Autrement dit, les inégalités sociales qui se sont mises en place à un moment ont un effet pérenne, par l'intermédiaire du niveau atteint à l'entrée dans l'année suivante (Duru-Bellat, 2003). La recherche en sciences de l'éducation (Demeuse, Crahay \& Monseur 2001, 2005, Crahay 2000, Demeuse \& Baye à paraître) a montré combien les structures éducatives pouvaient renforcer ces inégalités à tous les degrés de la scolarité, puisque tous les mécanismes de sélection explicites ou implicites instaurés ou non contrés par les systèmes éducatifs peuvent produire cet effet.

Parmi les mécanismes implicites, citons le regroupement des élèves dans des classes homogènes. Si cette pratique peut être favorable aux élèves les plus compétents, elle nuit gravement aux élèves les plus faibles en les plaçant dans des conditions souvent moins favorables -en termes de climat de travail, d'attentes des enseignants, de temps consacré effectivement à l'apprentissage. Or il s'agit là de facteurs d'efficacité pédagogique (Scheerens 2000) : ce que gagneraient les élèves faibles à la généralisation de classes hétérogènes est de l'ordre de deux fois plus que ce que les forts y perdraient. L'hétérogénéité des classes apparaît la solution la plus efficace pour optimiser les progrès des plus faibles, sans obérer proportionnellement les progrès des plus forts (Crahay 2000, Duru-Bellat 2003).

Certains pays de l'Union européenne se caractérisent aussi par de fortes différences entre établissements. Souvent, ces derniers sont bien plus inégaux que les élèves qu'ils accueillent (Coleman 1966, OCDE 2004, Grisay 2006). Un marché d'établissements de plus en plus diversifié est vecteur de ségrégation sociale. Les résultats de l'évaluation internationale PISA 2000 indiquent que la grande majorité des pays les plus performants en lecture font montre d'un grand degré d'homogénéité de performances entre établissements (OECD 2005), ce qui incite à poursuivre simultanément les objectifs d'équité et d'efficacité. 
Parmi les mécanismes explicites générant des inégalités sociales, citons la pratique du redoublement, encore possible dès le primaire et davantage encore au secondaire dans certains États-membres (Eurydice 2005). Dans sa synthèse sur l'échec scolaire, Crahay (1996) a montré que le redoublement est à la fois inefficace, puisqu'il ne permet pas aux élèves de combler leur retard, et inéquitable, car il est socialement et sexuellement marqué.

D'autres mécanismes structurels peuvent renforcer les inégalités à certains moments clés du système éducatif : les transitions (entre niveaux d'enseignement, filières, options...) sont susceptibles de renforcer les disparités entre élèves (Boudon 1973). En particulier, l'accumulation régulière des inégalités s'accélère lors du passage du primaire au secondaire inférieur (Sammons 1995, Duru-Bellat 2002), à tel point qu'en deux ans, autant d'inégalités sociales se créent que pendant toute la scolarité primaire (Duru-Bellat 2003). Par le jeu des filières et des options, les élèves de milieux défavorisés se voient offrir des programmes plus concrets. Les enseignants visent plus avec eux la motivation que les contenus, ont des attentes moins élevées à leur égard, des représentations plus négatives qui peuvent créer des "prophéties autoréalisatrices" ou effet Pygmalion (Rosenthal \& Jacobson 1971).

La Commission met en avant le caractère inefficace et inéquitable de filières précoces. La recherche confirme qu'une longue période de tronc commun avant toute sélection est moins sélective socialement (Crahay 2000, OCDE 2005) et évite le piège de la "démocratisation ségrégative" dénoncé par Merle (2000). Assurer la réussite de tous impose d'empêcher un système à plusieurs vitesses dans lequel certains élèves seraient amenés à poursuivre un enseignement valorisé tandis que d'autres, ayant accumulé du retard, seraient réorientés vers des écoles ou filières d'éducation spéciale distinctes. Cela pose à la fois le problème de la valorisation des filières, de l'orientation scolaire et de la place d'un enseignement spécialisé, séparé plutôt qu'intégré au système ordinaire.

À côté des pays scandinaves qui ont adopté des politiques volontaristes menant à la fois à une grande efficacité et une grande équité des résultats cognitifs, citons l'exemple récent de la Pologne, qui a modifié en profondeur ses structures éducatives, a allongé le tronc commun et réduit les inégalités sociales de performances à 15 ans, tout en élevant le niveau moyen (OCDE 2004).

Si toutes les différenciations implicites et explicites produisent de l'inégalité et souvent de l'inefficacité, c'est notamment parce qu'elles font intervenir le choix de parents inégalement informés et inégalement capables d'investir financièrement. Les moments de bifurcations et les paliers de la scolarité sont autant d'occasions d'(auto)sélection socialement différenciée de la part des écoles, des élèves et de leurs parents. La Commission ignore dans la discussion sur l'efficacité et l'équité les stratégies des familles en fonction de leur situation sociale et ne prend pas position sur la forte inégalité de la liberté de choix. Or ces stratégies 
doivent être prises en compte pour éviter un gâchis de talents. À cet égard, la proposition structurelle de la Commission, qui plaide pour la combinaison de l'autonomie locale et d'une responsabilisation centrale, doit être maniée avec beaucoup de prudence. En effet, "chaque fois que des possibilités de choix ont été introduites, elles sont utilisées avant tout par les familles de milieu aisé, notamment pour s'assurer que leur enfant sera bien scolarisé parmi les siens, dans une école pas trop hétérogène socialement ou ethniquement" (Duru-Bellat 2003, 60). Plus d'autonomie peut donc conduire à des choix stratégiques inégaux, selon la qualité d'information des usagers ou de formes d'autosélection familiale. La proposition d'augmentation de l'autonomie locale est interrogée par les comparaisons internationales du rendement scolaire. Celles-ci n'établissent pas de relation entre une plus grande autonomie des établissements et de meilleures performances des élèves (Duru-Bellat 2003, OCDE 2004). La supériorité de rendement des établissements autonomes et privés peut tenir de la composition socio-économique de leur public (OCDE 2005). Le renforcement de l'autonomie locale peut favoriser les disparités géographiques, offrant des conditions optimales pour que s'accentue une offre différenciée de filières, d'options, d'établissements inégalement prestigieux.

La Commission fait peu de recommandations sur les structures et les pratiques pédagogiques. Elle identifie un seul mécanisme structurel à la fois inefficace et inéquitable : la différenciation précoce par le biais des filières. Or les systèmes éducatifs sont des systèmes complexes, où les acteurs saisissent toute opportunité de distinction stratégique (Demeuse, Crahay \& Monseur 2001, 2005). Ces mécanismes, compréhensibles dans des systèmes discriminants où personne n'a intérêt à être du mauvais côté de la barrière éducative, doivent être pris en compte dans leur globalité pour favoriser l'efficacité et l'équité à l'échelle du système éducatif. Il est donc impératif de relever les mécanismes implicites et explicites de différentiation et d'anticiper les stratégies des acteurs, pour proposer des solutions efficaces.

Pour définir de bonnes pratiques, il importe d'identifier précisément les conditions dans lesquelles elles s'avèrent efficaces, tant pour accroître le niveau moyen des apprentissages que pour réduire les disparités entre élèves et limiter l'échec des plus faibles. Il convient de mettre à l'étude des protocoles qui permettraient d'identifier ces pratiques et leurs conditions de généralisation en impliquant des spécialistes des méthodes de recherche en éducation. La Commission devrait davantage soutenir des recherches observant et analysant des pratiques en classe et évaluant leurs résultats. Ce soutien implique aussi l'aide à la diffusion de telles pratiques. 


\section{Enseignement supérieur}

L es constats présentés dans le document de travail préparatoire de la Commission (SEC(2006) 1096) indiquent à juste titre que la massification de l'enseignement supérieur n'a pas été accompagnée de sa démocratisation : les jeunes issus de familles défavorisées sont toujours désavantagés en termes d'égalité d'accès et, par conséquent, d'obtention des titres correspondants. En outre, ceux qui accèdent à l'enseignement supérieur ne choisissent pas les orientations les plus rentables et ont tendance à abandonner plus vite en cas d'échec. Cette situation est autant préjudiciable en termes d'efficacité que d'équité, puisqu'il y a gaspillage de talents déterminé par des caractéristiques intrinsèques des individus.

La massification de l'enseignement supérieur étant un phénomène récent, ce constat peut paraître logique : ce sont les familles les plus favorisées qui continuent à se placer le mieux lorsque se généralise l'accès à un degré d'enseignement traditionnellement réservé à une élite. Même sans tenir compte du caractère récent de l'augmentation des effectifs de l'enseignement supérieur, ce phénomène est cohérent par rapport aux autres niveaux : les filières, options ou établissements les plus prestigieux sont sélectionnés et sélectionnent prioritairement un public favorisé. Le supérieur est pourtant désigné, plus que les autres, comme inéquitable, en raison de l'inéquitable distribution des ressources financières collectives qu'il mobilise. Le raisonnement est simple : la collectivité contribue au financement d'un bien éducatif dont les plus favorisés sont les bénéficiaires. Ce caractère injuste est seulement contredit si on tient compte des externalités positives que produisent les plus diplômés, notamment en termes d'innovation et de productivité. Ce constat peut être dressé, mutatis mutandis, pour le secondaire, mais la contribution personnelle des bénéficiaires est envisagée comme un moyen de rendre le système plus équitable. Ce qui peut se justifier davantage qu'à d'autres niveaux, si on tient compte de l'inégale répartition de leurs coûts relatifs (EGREES 2005, 175).

Si les constats de la Commission peuvent être partagés, les solutions proposées sont à interroger. La généralisation des droits d'inscription, même accompagnée de mesures d'aides aux moins nantis sous la forme de prêts risque de rendre l'enseignement supérieur encore plus inégalitaire. Les droits d'inscription introduisent des mécanismes de marché censés favoriser une saine pression au sein du système (SEC-2006 1096, 25) conduisant à une plus grande qualité d'enseignement et à une plus grande motivation à la réussite des élèves (augmentation de la présence aux cours, diminution des taux d'échec). Or, comme le reconnaît la Commission, les données manquent totalement en Europe pour étayer ces arguments alors que ce moyen peut entraîner les conséquences inverses. Ainsi, les pays de l'Europe du Nord, qui recouraient aux prêts dans l'enseignement supérieur ont constaté que les étudiants préféraient financer leurs études par du travail 
rémunéré. Le travail étudiant ayant tendance à prolonger la durée des études, ces pays ont finalement augmenté la part des bourses (Eurydice 1999).

Bayenet \& Demeulemeester (2005), dans leur revue de la littérature sur les politiques publiques de financement de l'enseignement supérieur dans les pays de l'OCDE, invoquent trois arguments pour préférer les bourses aux prêts et aux droits d'inscription : i) la non-existence d'études sur les effets de ces politiques en termes de choix de programmes et de durée des études, ii) la difficulté de déterminer l'incidence des obligations de remboursement et iii) l'exemple des pays de l'Europe du Nord confrontés aux résultats d'une politique privilégiant les prêts.

\section{Enseignement et formation professionnelle}

L a Commission regroupe dans une même section l'enseignement et la formation professionnels dispensés dans le cadre de l'enseignement secondaire ou dans celui des programmes pour adultes. Ce regroupement non conventionnel est intéressant, puisqu'il souligne la nécessité d'améliorer la qualité de toute formation liée au marché du travail. La Commission reconnaît que certaines formations professionnelles comportent des impasses, qu'il s'agisse de l'enseignement professionnel secondaire qui n'assure pas l'accès à l'emploi et à la formation ultérieure ou des programmes pour adultes à destination des moins qualifiés.

Pour améliorer la situation, la Commission propose deux démarches. La première consiste à renforcer les partenariats entre secteurs publics, privés et partenaires sociaux. La seconde est l'adaptation des programmes de formation aux besoins des employeurs. Si le dialogue entre le secteur du marché du travail et celui de l'enseignement et de la formation professionnels doit être conforté, il ne faut pas nourrir l'illusion que cette collaboration garantit une meilleure efficacité des systèmes d'éducation et de formation. Une trop forte adéquation des programmes de formation à des besoins ponctuels du marché du travail peut mener, en période de récession, à une forte inadéquation entre les compétences spécifiques acquises et les nouveaux besoins du marché du travail. En outre, la Commission souligne la difficulté à convaincre le secteur privé de financer la formation professionnelle, dès que celle-ci n'est pas en relation avec les besoins de l'entreprise. Cette réflexion plaide pour que le secteur public conserve un rôle important de promotion sociale des travailleurs, surtout les moins formés, de manière à permettre l'acquisition soit de compétences génériques, non directement en rapport avec l'emploi occupé, soit de compétences permettant d'acquérir une meilleure position sur le marché de l'emploi. 


\section{Action de l’Union européenne}

$\circlearrowleft$ ne collaboration internationale entre pays de l'Union européenne est indispensable. Leur destinée commune les pousse à collaborer à la définition des objectifs à atteindre et des méthodes pour les évaluer. Sur les méthodes, la Commission recommande l'échange de bonnes pratiques et reconnaît la responsabilité particulière des États-membres. Il est illusoire d'imaginer que des méthodes identiques produiront des résultats identiques dans des conjonctures historiques, culturelles, économiques et éducatives différentes. Pour être utile, la coopération entre États doit favoriser l'évaluation de pratiques prometteuses en envisageant leur efficacité, leur capacité à accroître l'équité et les conditions de leur transfert ou de leur généralisation.

La Commission voit dans l'amélioration de l'efficacité et de l'équité des systèmes éducatifs une source de mobilité des personnes. La mobilité est-elle entendue comme un résultat corollaire ou un objectif visé ? Ce n'est pas clair. S'il s'agit d'un objectif, il convient de le questionner. De quelle mobilité veut-on faire bénéficier les personnes : d'un palliatif des problèmes d'emplois régionaux qui ne résout rien ; d'une mobilité centrée sur l'emploi ; sur les échanges entre citoyens ? Dans tous les cas, il faut s'interroger sur la valeur ajoutée d'un investissement visant la mobilité. Il semble plus important de souligner celle d'un investissement centré sur le développement des compétences de base de tous, y compris et prioritairement des moins favorisés et des moins qualifiés. Il faut aussi interroger les conditions de la mobilité des étudiants, repenser la juste allocation des bourses d'échanges et leurs montants, car ces derniers ne permettent pas un accès équitable à des formations dans d'autres États-membres. Sans un rééquilibrage, le fossé entre des étudiants aisés et mobiles et des étudiants moins favorisés et moins mobiles bien qu'ayant accédé à l'enseignement supérieur va s'amplifier d'autant que la mobilité comptera pour l'emploi. La mise en place de centres d'excellence d'enseignement et de formation, plutôt qu'un service universel, accessible sur l'ensemble du territoire de l'Union, s'il n'est pas couplé à des aides réellement proportionnées aux coûts générés par cette mobilité, conduira à renforcer les disparités régionales et les écarts entre les individus selon leurs ressources propres.

La Commission conclut sur la priorité à donner à l'équité et à l'efficacité des systèmes éducatifs européens. Les remarques liminaires sur la nécessaire précision de la définition de ces concepts résonnent ici avec force. La culture d'évaluation et d'échanges doit être développée pour parvenir à ces objectifs. La Commission la prévoit uniquement dans le domaine de l'enseignement préprimaire, alors qu'elle devrait concerner tous les niveaux d'enseignement. L'enseignement et la formation doivent être conçus comme un ensemble en connexion avec les autres secteurs économiques et sociaux. Des politiques plus vastes visant 
à réduire les inégalités de condition de vie et de sécurité économique sont nécessaires pour augmenter l'égalité des chances (Shavit \& Blossfeld 1993). Une cohérence des politiques et de leur évaluation doit être recherchée. L'évaluation des politiques, la valorisation des travaux déjà menés sous l'égide de la Commission (par exemple les réseaux EURYDICE, le Network of experts in Social Sciences of Education and training -www.nesse.fr-, le GERESE -www.schoolequity.eu) doit être effectuée. Une politique ambitieuse et multilingue de valorisation des résultats de la recherche européenne en sciences de l'éducation doit être mise en place. Si l'économie de l'éducation constitue une discipline relativement structurée qui partage des méthodes communes, il n'en va pas de même des autres sciences sociales, dont les financements nationaux et internationaux sont en outre modestes. Il importe de soutenir le développement d'une approche cumulative, en mettant en place ou en soutenant le développement de centres de recherche européens sur l'éducation et la diffusion des savoirs, dans une perspective interdisciplinaire.

\section{Références bibliographiques}

BAYE A., DEMEUSE M., MONSEUR C. \& GOFFIN C. 2006 A Set of Indicators to measure Equity in 25 European Union Education Systems, Bruxelles, Rapport remis à la Commission européenne, Direction générale "Education et Culture"

BAYENET B., DEMEULEMEESTER J.-L. 2005 Politiques publiques de financement de l'enseignement supérieur : une tentative d'évaluation, in Demeuse M., Baye A, Straetent M.-H. \& Nicaise J. 2005 Efficacité et équité des systèmes d'enseignement et de formation, Bruxelles, De Boeck

BOUDON R. 1973 L'inégalité des chances. La mobilité sociale dans les sociétés industrielles, Paris, Armand Colin

COLEMAN J.S. 1966 Equal schools or equal students ? The Public Interest-4, 70-75

COMMISSION DES COMMUNAUTÉS EUROPÉENNES 2003 Investir efficacement dans l'éducation et la formation: un impératif pour l'Europe, Communication de la Commission (COM(2002)779 final), Bruxelles, CCE

COMMISSION DES COMMUNAUTÉS EUROPÉENNES 2006 Communication de la Commission au Conseil et au Parlement européen, Efficacité et équité des systèmes européens d'éducation et de formation (COM(2006)481 final), Bruxelles, CCE, http:// ec.europa.eu/education/policies/2010/doc/comm481_fr.pdf

COMMISSION OF THE EUROPEAN COMMUNITIES 2004 et sq. Commission staff working paper, Progress towards the Common Objectives in Education and Training. Indicators and Benchmarks, Bruxelles, CCE

CONSEIL EUROPÉEN 2000 Conclusions de la Présidence, Conseil européen de Lisbonne, 23 et 24 mars 2000 (DOC/00/8)

CRAHAY M. 1996 Peut-on lutter contre l'échec scolaire?, Bruxelles, De Boeck

CRAHAY M. 2000 L'école peut-elle être juste et efficace? De l'égalité des chances à l'égalité des acquis, Bruxelles, De Boeck 
CUNHA F., HECKMAN J., LOCHNER L. \& MASTEROV D. 2005 Interpreting the Evidence on Life Cycle Skill Formation. IZA Discussion Paper-1675. http://ssrn.com/ abstract $=766744$

DEMEUSE M. \& BAYE A. 2007 Medir y comparar la equidad de los sistemas edicativos en Europa, in Sancho Gargallo M.A. \& De Esteban Villar M. Libertad, Calidad Y Equidad en Los Sistemas Educativos (Buenas prácticas internacionales), Madrid, Comunidad de Madrid, Consejería de Educación y Fundación Europea Sociedad y Educación, 69-92

DEMEUSE M. \& BAYE A. (sous presse) Measuring and Comparing the Equity of Education Systems in Europe, in Soguel N. \& Jaccard P. (eds.), Educational Systems and the Challenge of Improving Results: Explaining and Enhancing Performance and Equity, Dordrecht, Springer

DEMEUSE M., BAYE A., STRAETEN M.H., NICAISE J. \& MATOUL A. (eds) 2005 Vers une école juste et efficace. 26 contributions sur les systèmes d'enseignement et de formation, Bruxelles, De Boeck Université

DEMEUSE M., CRAHAY M. \& MONSEUR C. 2001 Efficiency and Equity, in Hutmacher W., Cochrane D., Bottani N. In Pursuit of Equity in Education. Using international indicators to compare equity policies, Dordrecht, Kluwer, Academic Publishers

DEMEUSE M., CRAHAY M. \& MONSEUR C. 2005 Efficacité et équité dans les systèmes éducatifs. Les deux faces d'une même pièce ?, in Demeuse M., Baye A., Straeten M.H., Nicaise J. \& Matoul A. (eds) 2005 Vers une école juste et efficace. 26 contributions sur les systèmes d'enseignement et de formation, Bruxelles, De Boeck Université, 391-410

DURU-BELLAT M. 2002 Les inégalités sociales à l'école. Genèses et mythes, Paris, PUF

DURU-BELLAT M. 2003 Inégalités sociales à l'école et politiques éducatives, Paris, UNESCOInstitut international de planification de l'éducation

EGREES European Group for Research on Equity in Educational Systems 2005 Equity in European Educational Systems: a set of indicators, European Educational Research Journal, volume IV Number 2, 1-151

ELLEY W.B. ed. 1992 How in the World do Students Read?, Hamburg, Grindeldruck GMBH

EURYDICE 1999 L'aide financière aux étudiants de l'enseignement supérieur en Europe, Tendances et Débats, Questions clés de l'éducation-1, Bruxelles, Commission européenne

EURYDICE 2002 Chiffres clés de l'éducation en Europe, Bruxelles, Commission européenne

EURYDICE 2005 Chiffres clés de l'éducation en Europe, Bruxelles, Commission européenne

GRISAY A. 2006 Que savons-nous de l'“effet établissement” ?, in Chapelle G. \& Meuret D. éds, Améliorer l'école, Paris, PUF, 215-230

MERLE P. 2000 Le concept de démocratisation de l'institution scolaire : une typologie et sa mise à l'épreuve. Population-55-1, 15-50

MEURET D. 2001 School Equity as a Matter of Justice, in Hutmacher W., Cochrane D. $\&$ Bottani N. eds In pursuit of equity in education. Using international indicators to compare equity policies, Netherlands, Kluwer Academic Publishers

MULLIS I, MARTIN M., GONZALEZ E. \& KENNEDY A. 2003 PIRLS 2001 International Report. IEA's Study of Reading Literacy Achievement in Primary Schools in 35 Countries, Chesnut Hill, Boston College

MULLIS I.V.S., MARTIN M.O., GONZALES E.J. \& CHROSTOWSKI S.J. 2004a TIMSS 2003 International Mathematics Report. Findings From IEA's Trends in International Mathematics and Science Study at the Fourth and Eighth Grades, Chestnut Hill MA, Boston College 
MULLIS I.V.S., MARTIN M.O., GONZALES E.J. \& CHROSTOWSKI S.J. 2004b TIMSS 2003 International Science Report. Findings From IEA's Trends in International Mathematics and Science Study at the Fourth and Eighth Grades, Chestnut Hill MA, Boston College

OCDE 2004 Apprendre aujourd'hui, réussir demain. Premiers résultats de PISA 2003, Paris, OCDE

OECD 2001 Starting Strong. Early Childhood Education and Care, Paris, OECD

OECD 2006 Education at a Glance, OECD Indicators 2006, Paris, OECD

OECD 2005 School Factors Related to Quality and Equity. Results from Pisa 2000, Paris, OECD

REEZIGT G.J. ed. 2001 A framework for effective school improvement. Final report of the ESI project, Institute for Educational Research, University of Groningen, (Groningen) GION

ROSENTHAL R. \& JACOBSON L. 1968 Pygmalion in the classroom: teacher expectation and pupils' intellectual development, New York, Holt, Rinehart and Winston

SAMMONS P. 1995 Gender, ethnic and socio-economic differences in attainment and progress : a longitudinal analysis of student achievement over 9 years, British Educational Research Journal-21-4, 465-485

SCHEERENS J. 2000 Improving school effectiveness, Paris, UNESCO

SHAVIT Y. \& BLOSSFELD H. 1993 Persistent Inequality, Boulder, Westview Press

SLAVIN R.E. \& FASHOLA O.S. 1998 Show Me the Evidence! Proven and Promising Programs for America's Schools, Thousand Oaks, Corwin Press

WANG M.C., HAERTEL G.D. \& WALBERG H.J. 1998 Models of Reform: Acomparative Guide. Educational Leadership, April

WÖBMANN L. \& SCHÜLTZ G. 2006 Efficiency and Equity in European Education and Training Systems, http://ec.europa.eu/education/policies/2010/doc/eenee.pdf 